\title{
Toward a General Model for the Evolution of DNA Replication in Three Domains of Life
}

\author{
R. Retkute * \\ School of Biosciences, Sutton Bonington Campus, University of Nottingham, LE12 5RD, UK \\ School of Mathematics, University Park, Nottingham, University of Nottingham, NG7 2RD, UK

\begin{abstract}
Nothing is more fundamental to life than the ability to reproduce and duplicate the information cells store in their genomes. The mechanism of duplication of DNA has been conserved from prokaryotes to eukaryotes. The aim of the study was to quantify which evolutionary forces could produce the pattern of genome replication architecture observed in present-day organisms. This was achieved using an evolutionary simulation, combining random genome sequence shuffling, mutation, selection and the mathematical modeling of DNA replication. We have found parameter values which explained evolutionary pressures of DNA replication in E.coli, P.calidifontis and S. cerevisae. Surprisingly, the results of the evolutionary simulation suggests that for a fixed cost per replication origin it is more advantageous for genomes to reduce the number of replication origins under increasing uncertainty in origin activation timing.
\end{abstract}

Keywords and phrases: DNA replication, modeling biological evolution, mutation, selection, fitness

Mathematics Subject Classification: 92B05, 92D15, 92D20

\section{Introduction}

Nothing is more fundamental to life than the ability to reproduce. This ability begins with the simple fact that all living organisms are able to duplicate the information they store in their genomes by the process of DNA replication. A mathematical theory of evolution indicates that the selection for replication, and therefore the benefits of being able to self-replicate, allowed transition from prebiotic chemistry to life in the primordial soup of information molecules [35].

The main principles of the replication process are conserved across all three domains of life (the bacteria, the archaea and the eukarya), therefore many of the biochemical mechanisms that regulate DNA replication are similar between different living organisms. Schematics of the replication process is shown in Fig.1 (a). DNA synthesis begins at specific sites along a genome called replication origins. Trans-acting initiators recognize replication origin sequences where prereplicative complexes are assembled; initiator proteins bind to the DNA and pry the two strands apart so that a single-stranded DNA template becomes ready for the loading of DNA helicase [24]. Once the double helix is opened, it attracts a group of proteins that carry out DNA replication. Genome in the process of being replicated contains Y-shaped junctions

*Corresponding author. E-mail: renata.retkute@nottingham.ac.uk 
(a)
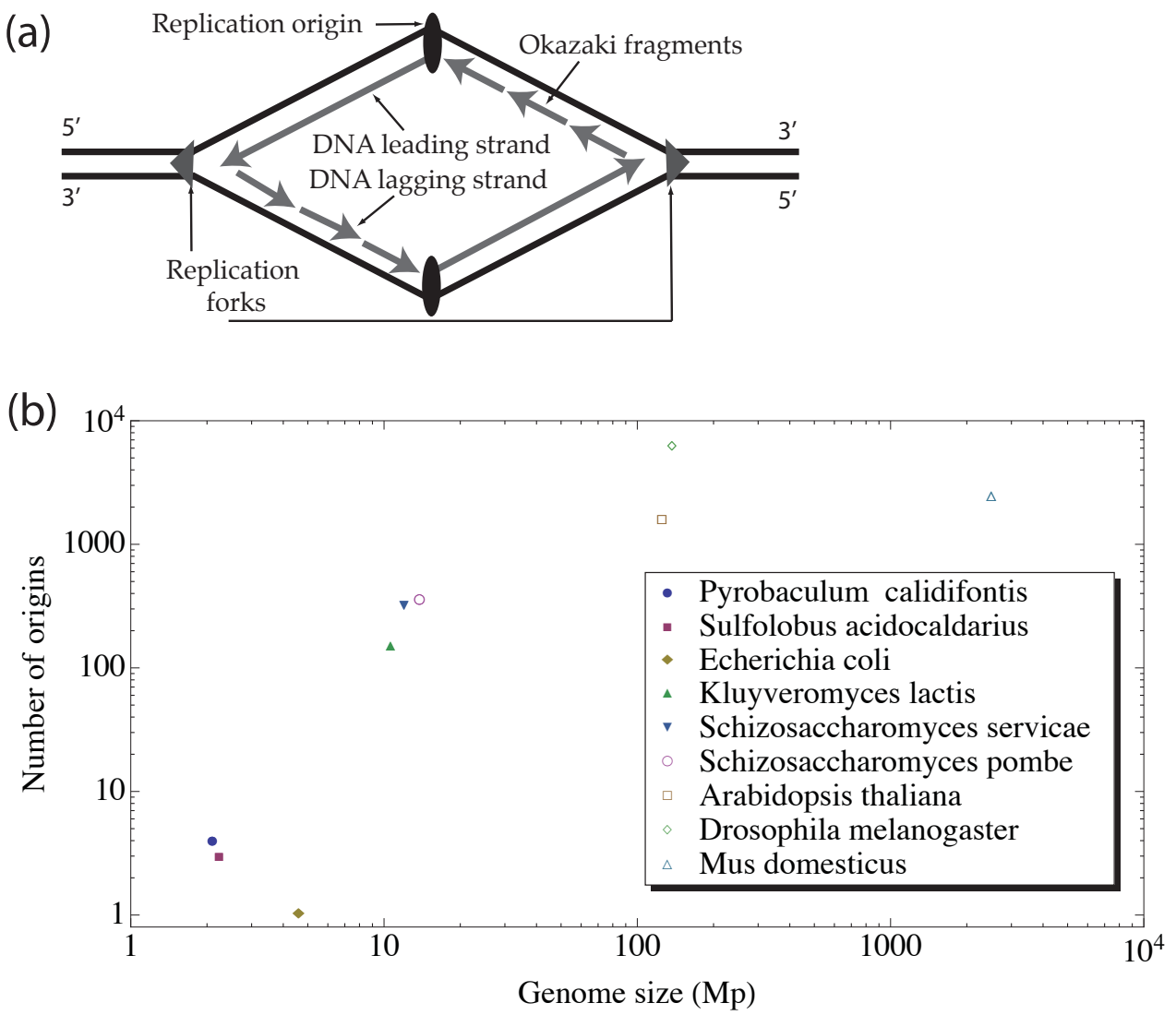

FiguRE 1. (a) Schematics of the replication process, which is conserved in three domains of life: DNA synthesis starts at a replication origin and always progresses in the 5'-3' direction, with one strand (the leading strand) synthesized continuously, and one strand (the lagging strand) synthesized discontinuously by Okazaki fragments. (b) Genome sizes and the number of replication origins. Sources: Pyrobaculum calidifontis [37]; Sulfolobus acidocaldarius [27]; Escherichia coli [45]; Kluyveromyces lactis [14,25]; Schizosaccharomyces servicae [43]; Schizosaccharomyces pombe [14,19]; Arabidopsis thaliana [10,14]; Drosophila melanogaster $[7,14]$; Mus domesticus $[7,14]$.

called replication forks. Two replication forks are formed at each replication origin, and they move away from the origin in opposite directions, unzipping and duplicating the DNA as they go, though DNA polymerases can synthetize DNA only in one direction [24]. Replication is continuous on the leading strand and discontinuous on the lagging strand, replicated by short segments called Okazaki fragments. Replication forks terminate when the fork reaches the telomeric or termination region of the chromosome, or two forks traveling in a head-on direction collide. A portion of DNA replicated from any particular replication origin is termed a replicon.

Although all three domains of life use the same mechanism to replicate their genomes, there are differences in genome structure and the strategies that they employ to ensure appropriate genome duplication [42]. First, bacterial and archaeal genomes are composed of a single chromosome, while eukaryotic genomes are partitioned into multiple chromosomes. Differences are also determined by the initiation 
of DNA synthesis in space: in bacterial genome duplication is initiated at a single locus, while in some archaeal and all eukaryotic cells DNA replication can initiate at multiple replication origins. As the time required to complete the duplication of daughter chromosomes varies between species, the number of replication origins becomes an important issue in genome replication.

The number of replication origins ranges from one in bacteria [45] to a few hundred in yeast [43] to tens of thousands in metazoan cells [7]. Figure 1 (b) shows the genome sizes and number of replication origins for a variety of organisms: bacteria Escherichia coli (0.21 origins per $\mathrm{Mb}$ ) [45]; archaeas Pyrobaculum calidifontis (1.9 origins per $\mathrm{Mb}$ ) [37] and Sulfolobus acidocaldarius (1.34 origins per Mb) [27]; eukaryotes Kluyveromyces lactis (14.0 origins per Mb) [14,25], Schizosaccharomyces servicae (26.0 origins per Mb) [43], Schizosaccharomyces pombe (25.2 origins per Mb) [14,19], Arabidopsis thaliana (12.3 origins per Mb) [10,14], Drosophila melanogaster (45.1 origins per Mb) [7, 14] and Mus domesticus (0.97 origins per $\mathrm{Mb}$ ) $[7,14]$. It can be seen that there is a complex relationship between genome size and number of replication origins. For example, the E.coli genome has only one replication origin and $4.6 \mathrm{Mb}$ genomic material, while P.calidifontis genome size is $2.1 \mathrm{Mb}$ and it has 4 replication origins.

Despite progress in characterizing the distribution of replication origins along genome in various model systems, our understanding of the underlying mechanism that establishes replication organization has remained sparse. In this study we are going to use an evolutionary simulation, combining random genome sequence shuffling, mutation, and selection together with the mathematical modeling of DNA replication to quantify which evolutionary forces could produce the number and positions of replication origins observed in present-day species. We will compare our results with the trends observed in the bacteria E.coli, archaea P.calidifontis and eukaryote $S$. cerevisae chromosome IV.

\section{Methods and materials}

To study the effect of evolutionary pressures sculpturing replication in different organisms, we used an evolutionary simulation framework. The scheme of the simulation is shown in Figure 2 (a). The simulation framework was executed using following steps. We generated $N$ artificial genomic sequences by shuffling the observed sequence of the length $L b p$ while maintaining the genome's overall nucleotide composition. We set $N=10^{3}$. The genome sequences were then analysed and locations containing the consensus sequence were found, which gave a number of replication origins, $n$, and their positions, $x_{i}, i=1 . . n$. Origin replication times, $t_{i}$, were drawn from a random distribution. Then each genome was duplicated once and a copy was mutated according to a mutation rule. New genome sequences were analysed and a list of replication origins was updated. Finally, the fitness was evaluated for each of $2 N$ genomes, and $N$ genomes with the highest fitness value were selected for next generation. We ran the evolutionary simulation for 5000 generations.

The DNA molecule is a polymer made up of four nucleotides: T, C, A, and G, but their abundance in genomes is not uniform. Evolution of nucleotide compositional asymmetry has been influenced by various cellular processes, such as replication [2,3], transcription [18], translation [13], recombination [9], chromatin conformation [40], or methylation [48]. Nucleotide compositional asymmetry due to replication is based on the difference in mechanisms of the synthesis in the leading and lagging strands with the leading strand being more G+T-rich. Nucleotide composition bias has been used to predict replication origin sites in bacterial and archaeal chromosomes [52] and human genome [6]. A model linking replication and transcription activity to nucleotide compositional asymmetry showed that transcription-associated asymmetry changes sign with gene orientation and increases in magnitude with transcription rate while replication-associated asymmetry is proportional to a difference between the fraction of forks moving rightward to leftward $[2,3]$.

The replication-timing program may be a driving force in the copy number and single-nucleotide polymorphism diversity that is observed within and between species [11]. A strong correlation between the time at which a region of the chromosome is replicated and its mutation rate has been identified in budding yeast, while the average GC content has not correlated with mutation rate [23]. Mutation rate has been found to be elevated in the late replicating regions in D. melanogaster [51] and the rate 

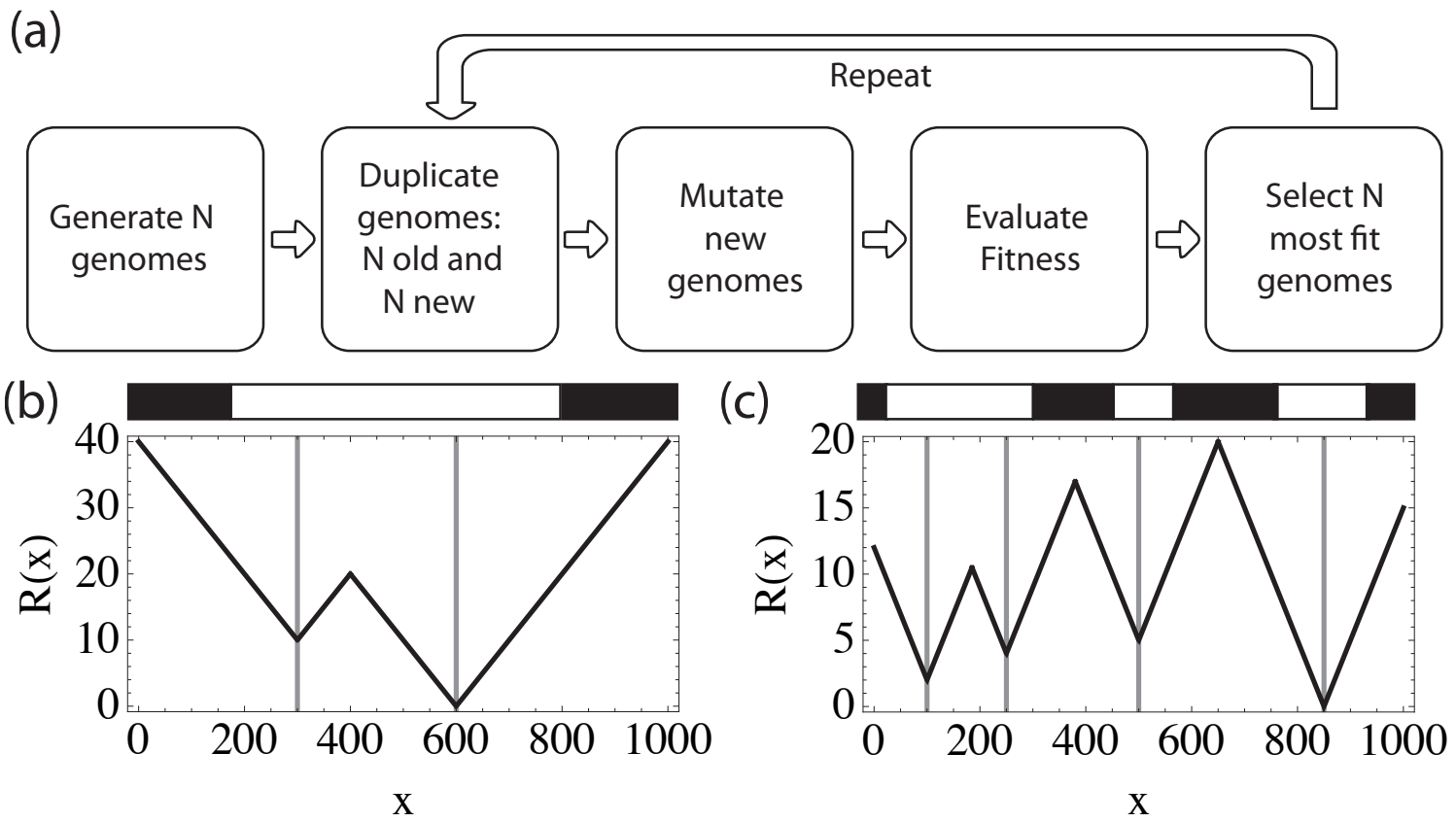

FiguRE 2. (a) The evolutionary simulation scheme. (b)-(c) DNA replication of virtual chromosomes: (b) with two replication origins located at 300 and 600 and activation times 0 and 10 minutes, (c) with four replication origins located at 100, 250, 500, and 850, and activation times 2, 4, 5, and 0 minutes. Parameter values: $L=1000 \mathrm{bp}$ and $v=10 \mathrm{~kb} / \mathrm{min}$. Dark rectangles above the graphs indicate late replicating regions and white rectangles indicate early replicating regions.

of evolutionary divergence between human-chimpanzee genomes increased by $22 \%$ during the temporal course of replication [47].

The repertoire of naturally occurring changes in genome is very rich and ranges from point mutations, insertions and deletions to duplications of genes and whole genomes or other large scale genome rearrangements [30]. In our evolutionary simulation, we considered only point mutations and we assumed that nucleotides mutate independently from each other. The mutation rule was executed as follows: genomic sites were divided into early and late replicating regions. We assumed that nucleotides within early replicating region could mutate with probability $\eta$, and nucleotides within late replicating region could mutate with probability $\mu$, where $\eta<\mu$. A random position along the genome was chosen together with a second position within the same type of replication characteristic (early or late replicating region). Their nucleotide content was exchanged depending on the mutation probability. In this way the nucleotide composition of genome was maintained during all generations.

Experiments showed that the mutation rate varied by an order of magnitude across $S$. cerevisae Chromosome VI: early replicating regions had a low per base mutation rate and late replicating regions had a high per base mutation rate [23]. The rates of spontaneous mutation per nucleotide site per cell division have been found to be quite similar across a variety of organisms: $2.6 \times 10^{-10}$ in E.coli, $3.3 \times 10^{-10}$ in S. cerevisae, $1.6 \times 10^{-10}$ in A.thaliana and $1.3 \times 10^{-10}$ in D.melanogaster [31]. Therefore, we have set $\eta=1 \times 10^{-10}$ per-base pair-per-generation and $\mu=1 \times 10^{-9}$ per-base pair-per-generation for all evolutionary simulations.

The next step was to define the positions of replication origins. Comparative analysis of replication origin distribution in different organisms showed that the organization of replication origins along genome is site-specific, but flexible: replication origins in S.cerevisiae share a 12-17-bp specific consensus sequence, 
while in multicellular eukaryotes they lack sequence specificity [33]. Therefore we have used an artificial consensus sequence defined by $l$ repeats of the nucleotide A. Replication origins were positioned at a middle of consensus sequence.

Modeling of genome-wide replication kinetics has revealed how activation of replication origins during synthesis phase governs replication timing profiles $[1,4,12,16,28,29,50]$. Parameters determining DNA replication are the number, positions and activation times of replication origins. Although replication origins can have a distinct activation distributions [12,50], and their functionality is shaped by genetic and epigenetic processes [33], we assumed that all replication origins were activated according to a continuous uniform distribution, $t_{i} \sim U\left[T_{\min } . . T_{\max }\right]$. We have set $T_{\min }=0$, while value of $T_{\max }$ indicated the uncertainty in origin activation timing. We assumed that replication forks moved bidirectionally with a constant velocity $v k b \mathrm{~min}^{-1}$.

Replication time at any given chromosome position, $x$, was calculated as:

$$
\begin{aligned}
R(x)= & \min _{i}\left(t_{i}+\frac{\left|x-x_{i}\right|}{v}\right), \\
R(x)= & \min _{i}\left(t_{i}+\frac{\left|x+k L-x_{i}\right|}{v}\right), \\
& x=1 . . L ; i=1 . . n ; k=-1,0,1 .
\end{aligned}
$$

In organisms with a linear chromosome DNA replication can be described by Eq.(2.1) and in organisms with a circular chromosome - by Eq.(2.2). Total replication time was defined as the maximum time required to replicate a whole chromosome:

$$
R_{T}=\max _{1 \leq x \leq L} R(x) .
$$

Replication of a virtual chromosome with $L=1000 \mathrm{bp}, v=10 \mathrm{bp} / \mathrm{min}$ and two replication origins located at $x_{1}=300$ and $x_{2}=600$ is shown in Fig.2(b)). For origin activation times $t_{1}=0 \mathrm{~min}$ and $t_{2}=10 \mathrm{~min}$, the following properties describe replication dynamics: (a) four replication forks are assembled; (b) forks terminate at three locations $-x=0 b p, x=400 b p$ and $x=1000 b p$; and (c) total replication time is 40min. For a virtual chromosome with four replication origins located at $x_{1}=100, x_{2}=250, x_{3}=500$, and $x_{4}=850$ (fig.2(c)), replicon sizes are smaller and total replication time is shorter $\left(R_{T}=20 \mathrm{~min}\right)$. Dark rectangles above the graphs indicate late replicating regions $\left(0 \leq t<R_{T} / 2\right)$ and white rectangles indicate early replicating regions $\left(R_{T} / 2 \leq t \leq R_{T}\right)$.

Activation of replication origins is a costly process requiring mobilization of cellular resources - an assembly of replication machinery. Hence we expect that the selection step will drive replication evolution towards a level at which the cost of sustaining origins is balanced by their benefit on fitness. Therefore, we define fitness as a function of the time required to replicate a whole chromosome and the cost of sustaining a particular number of origins:

$$
w= \begin{cases}-100, & \text { if } n=0, \\ \frac{L / v}{R_{T}}-\kappa n, & \text { otherwise. }\end{cases}
$$

If no replication origin exists $(n=0)$, cells can not duplicate their genomic content and so are prevented from cellular division and contribution to the next generation. Replication is the most vulnerable period of the cell cycle to accumulate genomic instability and DNA damage [11]: deletion of the earliest origin on yeast chromosome VI increased the mutation rate by $30 \%$ [23], while the frequency of intergenic mutations was significantly higher in late DNA replication regions in human cancer genomes [26]. In bacteria, coorientation of replication and transcription has been linked to a selection for speedy replication, as slower replication imposed a small but significant competitive disadvantage [46]. The ratio $\frac{L / v}{R_{T}}$ indicates how much faster a genome is replicated from a given set of replication origins compared to replication by a single fork. 
TABLE 1. Genomes and their properties.

\begin{tabular}{|l|c|c|c|c|c|}
\hline Genome & NCBI identifier & Length & No of origins & Fork velocity & G+C \% \\
\hline E.coli & 26111730 & $5,231,428 \mathrm{bp}$ & 1 & $65 \times 10^{3} \mathrm{bp} / \mathrm{min}$ & 51 \\
P.calidifontis & 126458628 & $2,009,313 \mathrm{bp}$ & 4 & $2100 \mathrm{bp} / \mathrm{min}$ & 57 \\
S. cerevisae, chrom. IV & 330443520 & $1,531,933 \mathrm{bp}$ & 71 & $2000 \mathrm{bp} / \mathrm{min}$ & 38 \\
\hline
\end{tabular}

Conversely, a large number of replication origins will ensure faster replication, but could lead to the exhaustion of cellular resources. We introduced a single parameter to describe the cost of replication, $\kappa$. Parameter $\kappa$ depends on the environmental and developmental needs of an organism [32] and serves as a penalty arising from each additional replication origin and is a limiting factor for genome replication.

We ran the evolutionary simulation scheme for E.coli, P.calidifontis and S. cerevisae chromosome IV. Complete genomes were downloaded from the National Center for Biotechnology Information (NCBI; http://www.ncbi.nlm.nih.gov/) in FASTA nucleotide format. We calculated the following measures of nucleotide compositional asymmetry: GC skew $S_{G C}=\frac{G-C}{C+G}$ (the excess of G over C normalised to the GC content), AT skew $S_{A T}=\frac{A-T}{A+T}$ ( the excess of A over T normalised to the AT content) and total skew $S=\frac{G-C+A-T}{C+G+A+T}$ (the sum of excess of $\mathrm{G}$ over $\mathrm{C}$ and A over T). Genomes, their NCBI identifiers, lengths, number of origins, replication fork velocities and percents of a total $\mathrm{G}+\mathrm{C}$ content are given in Table 1.

\section{Results}

Nucleotide compositional profiles were calculated within overlapping windows of $100 \mathrm{~kb}$ at a $1 \mathrm{~kb}$ resolution. The GC, AT and total skews are shown for the E.coli genome (Fig.3 (a)), P.calidifontis genome (Fig.3 (d)), and S. cerevisae chromosome IV (Fig.3 (g)). Horizontal grey lines indicate the observed positions of replication origins. Overall, E.coli and P.calidifontis have a higher total $G+C$ content compared to $S$. cerevisae (Table 1). Nucleotide compositional asymmetry can only be observed for E.coli: the GC skew changes sign from a positive on the lagging strand to a negative on the leading strand, the $A T$ skew and total skew change sign from a negative on the lagging strand to a positive on the leading strand. There is no visible nucleotide compositional asymmetry in the vicinity of the replication origins of P.calidifontis and $S$. cerevisae, although nucleotide skew profiles irregularly crossed the x-axis. A technique based on detrended DNA walks failed to detect replication related compositional bias in $S$. cerevisiae chromosomes, except in the subtelomeric regions [17].

For the evolutionary simulation scheme, described in detail in the methods and materials section, we have varied values of $l, T_{\max }$ and $\kappa$ in order to find the conditions which would explain the evolutionary pressure of DNA replication in E.coli, P.calidifontis and S. cerevisae.

Replication origin locations have been predicted for more than 2000 bacterial genomes [15] and a considerable variability has been observed in both the length of initiator recognition sequences (ranging from 100 to $1000 \mathrm{bp}$ ) and their nucleotide composition [24]. The genome of E. coli has a single replication origin, usually termed oriC. A pair of replication forks duplicate the whole genome by traveling in opposite directions until the replication forks arrive at the terminus site [41]. The existence of terminus sites allows us to interpret the E.coli genome as linear, with the replication origin located at the center of the genome [41]. Under low grow it takes about 40 minutes to replicate the E.coli genome [44], which gives $v=65 \mathrm{~kb} / \mathrm{min}$. We ran the evolutionary simulations with $l=8,9,10 \mathrm{bp}$, and found that for $l<10 \mathrm{bp}$ the algorithm did not converge in $10^{3}$ steps, giving $l=10 \mathrm{bp}$. Figure 3 (b) and (c) shows the results of evolutionary simulations for $\kappa=1.9$ and $T_{\max }=0$. About $85 \%$ of the predicted outputs only had a single replication origin positioned in the interval $[0.47 / L, 0.52 / L]$. This is in agreement with the observed position in the linearised E.coli genome. Evolutionary simulations with $T_{\max }=5$ gave that $100 \%$ of predicted genomes had a single replication origin. 


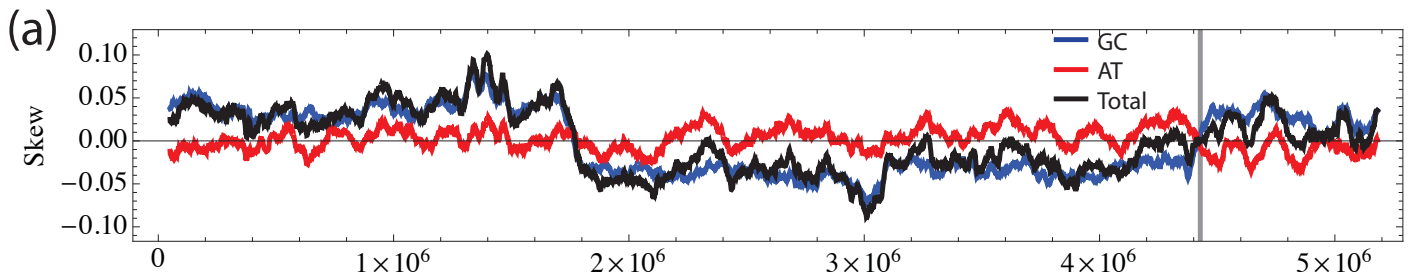

(b)

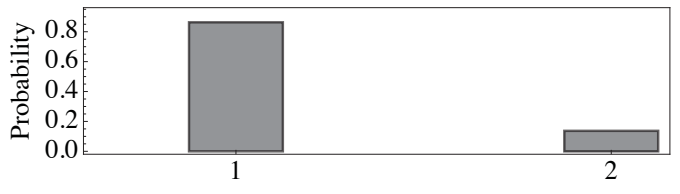

(d) Number of origins (c)

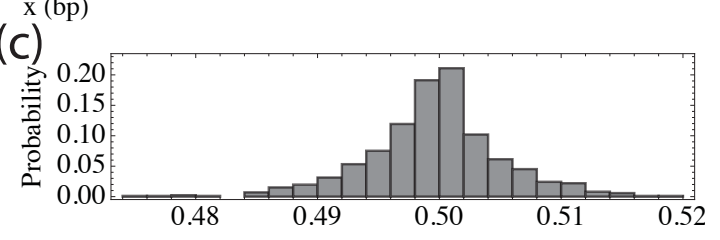

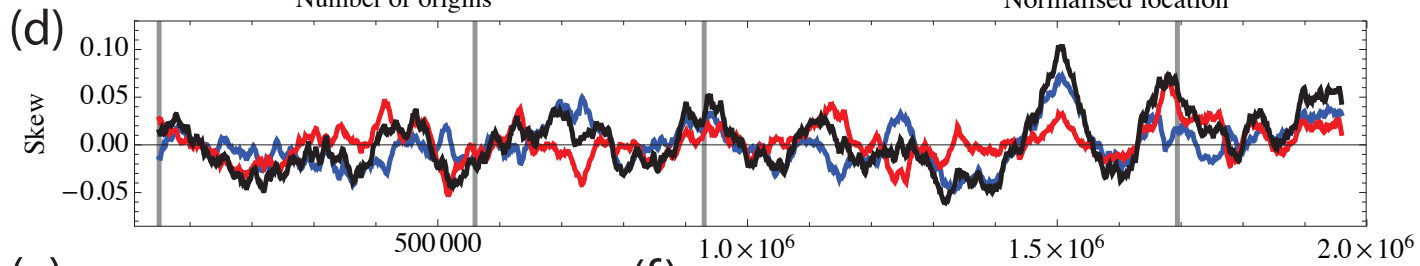

(e)

(f) $\mathrm{x}(\mathrm{bp})$

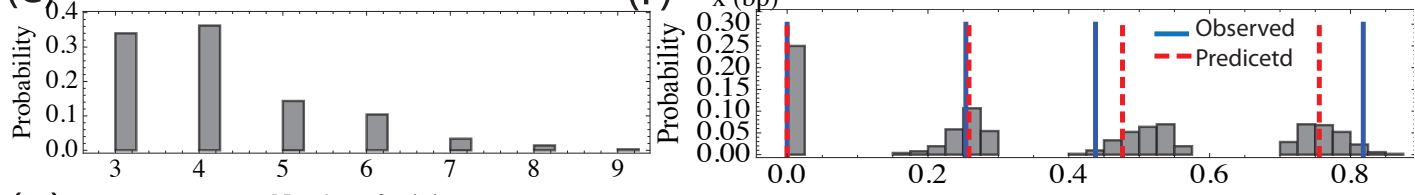

(g)

Number of origins

Normalised location

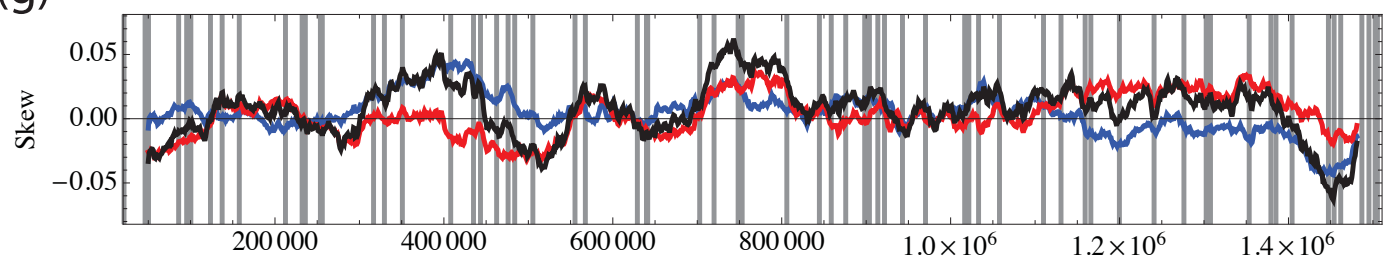

(h)

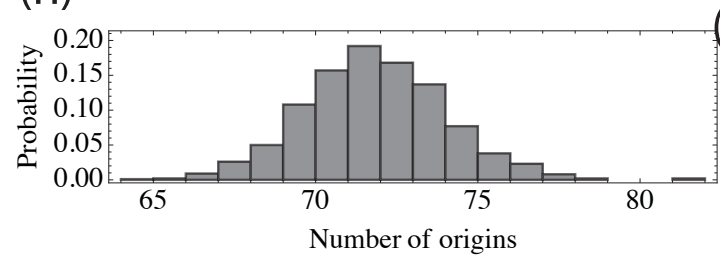

$\mathrm{x}(\mathrm{bp})$

(i)

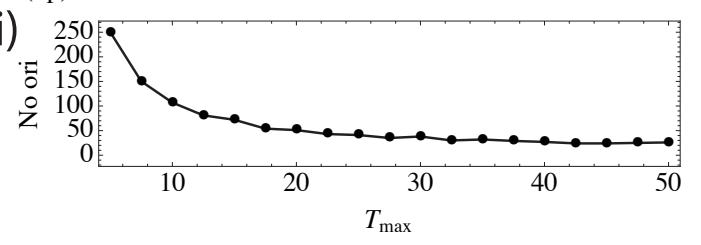

Figure 3. (Color online) Nucleotide compositional profiles and results for the evolutionary simulations: (a)-(c) E.coli, (d)-(f) P.calidifontis and (g)-(j) S.cerevisae chromosome IV. Nucleotide skews $S_{G C}$ (red line), $S_{A T}$ (blue line) and $S$ (black line) were calculated within overlapping windows of $100 \mathrm{~kb}$ at each $1 \mathrm{~kb}$ resolution and values were asigned to the position of a center of the window. Horizontal grey lines in nucleotide compositional profiles indicate the observed positions. Positions along chromosomes are normalised with respect to the length, $L$.

Archaeal genomes have a circular chromosome with one or more origins, while archeael DNA replication machinery contains a mixture of attributes found in bacterial and eukaryotic cells [24]. Archaeon P.calidifontis has four replication origins, the highest number detected in any prokaryotic organism [37]. 
Relative positions of the replication origins are $0 \mathrm{~kb}, 500 \mathrm{~kb}, 900 \mathrm{~kb}$, and $1600 \mathrm{~kb}$. Fork velocity was estimated to be $65 \mathrm{bp} \mathrm{s} s^{-1}$ [37], or $2.1 \mathrm{~kb} \mathrm{~min}^{-1}$. We have used $l=8 \mathrm{bp}$ as this showed a good rate of convergence in the evolutionary simulations. Marker frequency analysis in [37] suggested that initiation of replication at all origins occurred within a short time interval. Figure 3 (e) and (f) shows the results of the evolutionary simulation for $\kappa=1.4$ and $T_{\max }=5$. The distribution of the number of replication origins indicates that about $40 \%$ of genomes had four replication origins, about $35 \%$ had three replication origins and a small proportion had 5-9 replication origins. Figure 3 (f) shows the distribution of positions of predicted replication origins normalized along the length of the genome for the outputs which had exactly four replication origins predicted. For each predicted genome the start of the genome has been moved to a location of the first origin. Horizontal blue lines indicate locations of the observed replication origins and red dashed lines show the predicted positions of the replication origin. Although there is an overall agreement between observed and predicted locations, there is an accumulation of discrepancy towards the last origin.

Specific consensus sequences of replication origins have been identified only in the eucaryote $S$. cerevisiae. This $11 \mathrm{bp}$ sequence $[5-/(\mathrm{A} / \mathrm{T}) \mathrm{TTTA}(\mathrm{T} / \mathrm{C})(\mathrm{A} / \mathrm{G}) \mathrm{TTT}(\mathrm{A} / \mathrm{T})-3]$ can be found in 12000 locations across its genome [24], but only about 300 of them serve as replication origins [43]. We ran the evolutionary simulation on the $S$. cerevisiae chromosome IV, as this is the largest chromosome and it has a comparable size to the genomes of E.coli and P.calidifontis. Although each replication origin in $S$. cerevisiae has a particular window when it is activated in different cells [12,50], we assumed that all replication origins have identical activation distributions. Fork velocity in $S$. cerevisiae has been found to be about $2 \mathrm{~kb} \mathrm{~min}^{-1}[12,50]$. We set $l=8 \mathrm{bp}$. Figure $3(\mathrm{~h})$ shows the distribution of a number of replication origins for parameters $\kappa=0.25$ and $T_{\max }=15$. The median of the predicted distribution is 71 , which is equal to the observed number of replication origins. Next, we analysed how results of the evolutionary simulations depend on the uncertainty in activation timing. We set $\kappa=0.25$ and varied values of $T_{\max }$ from 5 till 50 minutes. Figure 3 (i) shows the median number of replication origins as a function of $T_{\max }$. It can be seen that the number of replication origins is reduced with increasing uncertainty in origin activation timing.

\section{Discussion}

Timely and orderly replication is essential to guarantee the faithful transmission of genetic information. In this work we have used evolutionary simulations in order to get insight into a possible mechanism underlying DNA replication in different species.

The interplay between selection, its efficiency, and mutation rates can result in a variety of scenarios. Replication origins can be positioned at specific locations along a genome after random shuffling of genome sequences or as a result of a mutation during evolution. The continuous selection provides means for retaining those genome configurations (number of the replication origins and their positions) which can balance the benefit of faster replication and the cost required to maintain this particular configuration. Parameters of evolutionary simulations could be divided into two groups: fixed (nucleotide composition, mutation rates, length of the genome and replication fork velocity) and variable (length of replication initiation sequence, cost per replication origin and maximum origin activation time). We obtain the following values of the cost per replication origin: $\kappa=1.9$ (bacteria E.coli), $\kappa=1.4$ (archaea P.calidifontis) and $\kappa=0.25$ (eukaryote $S$. cerevisae chromosome IV). These values indicate that the cost per replication origin is closer between bacterial and archaeal genomes than between bacteria and eukariotic genomes or archaeal and eukariotic genomes, as can be expected from the differences in replication initiation complexes [24]. S. cerevisae is able to sustain a high number of replication origins by making them less demanding in terms of the required limiting factors.

There is experimental evidence that closely related species can share a genetic information encoded at replication origins. For example, replication origins in 15 completely sequenced haloarchaeal genomes were predicted by identification of specific sequences that are associated with orc/cdc6 genes and comparative analysis showed that different species of halophilic archaea shared a few conserved origins [53]. The 
analysis of genome replication in four species within the Saccharomyces sensu stricto clade also showed that the locations of some replication origins were conserved [34]. In our proposed evolutionary simulation algorithm, we have ignored phylogenic dependencies and ran evolutionary simulations for each species separately with an artificial consensus sequence defined by $l$ repeats of the nucleotide A. The length of sequence was determined for each organism by observing a convergence of simulations (changes in mean fitness value). We found the following values: $l=10 b p$ (bacteria E.coli), $l=8 b p$ (archaea P.calidifontis) and $l=8 b p$ (eukaryote $S$. cerevisae chromosome IV). We found that the evolutionary simulations required $l=10 b p$ for consensus sequence containing repeats of the nucleotide $\mathrm{G}$ or $\mathrm{C}$, which can be explained by a high value of total $\mathrm{G}+\mathrm{C}$ content of P.calidifontis.

Surprisingly, the results of the evolutionary simulations suggest that for a fixed cost per replication origin it is more advantageous for the genome to reduce the number of replication origins with increasing uncertainty in origin activation timing. The distribution of the predicted number of replication origins for the $S$. cerevisae chromosome IV indicated that the median number of replication origins was continuously reduced when $T_{\max }$ increased: it was 230 at $T_{\max }=5 \mathrm{~min}$, and reached 25 at $T_{\max }=50 \mathrm{~min}$. Keeping in mind that the length of the $\mathrm{S}$ phase in $S$. cerevisae is roughly $60 \mathrm{~min}$ [50], a large value of $T_{\max }$ means that replication origins can activate almost at anytime during the $\mathrm{S}$ phase.

Next, we will look at the shortfalls of the evolutionary simulation algorithm we used and what could be directions for improvement. First, a limitation of our model is that we considered only the simplest process for genome change - point mutations, while genomes evolve through a variety of mechanisms. Point mutations can not account for large changes in evolution, these changes are made possible by horizontal gene transfer in prokaryotes [41] and by gene duplication or polyploidy (duplication of the entire genome) in eukaryotes [36]. For example, comparative genomic analysis indicated that S.cerevisiae actually arose from ancient whole-genome duplication either by endo-duplication (auto-polyploidy) or the fusion of two close relatives (allo-polyploidy) followed by a large number of deletion and rearrangement events which have shuffled the duplicated chromosomes [21]. Other mechanisms, which would be of interest to include in the simulation of genome sequence dynamics as a separate process or joint processes, could be a small insertion or deletion at the nucleotide level, segmental duplication or deletion, chromosome fission, insertion of mobile elements or introns and chromosome fusion [30].

When formulating the simulation procedure, we considered the activation time for all origins to follow a continuous uniform distribution. Activation time can be influenced by multiple cellular processes, such as nucleosome positioning, histone modifications, three-dimensional organization of the nucleus [33], and even the composition of neighbor DNA sequences can advance origin activation time [38]. Also, origin activation has a stochastic component: there are differences not only when replication origins activate, but also which of the replication origins are used in different cells in a population. Therefore, our results give the upper limit of the cost of replication, $\kappa$.

In mammals the mechanisms coordinating replication are similar to prokaryotes and unicellular eukaryotes - initiator binding, determination of replication timing, and selection of which replication origins will be activated, each occurring independently at distinct times during cell cycle [20]. The profiling of replication timing has showed a clear segmentation of chromosomes into $M b$ sized domains with a defined temporal sequence [39]. The genome-wide locations of replication origins in human cells have been mapped using deep sequencing of short nascent strands, which allowed the identification of a consensus motif that can predict the position of DNA replication origins [5]. The metazoan replication origins were largely interchangeable in mice and flies [8]. Also, the replication timing was found to be conserved between human and mice, indicating that large chromosomal domains were shuffled by evolution while conserving the large-scale nuclear architecture of the genome [49]. It would be possible to extend the evolutionary simulation algorithm we proposed to the mammalian organisms by varying the interval $\left[T_{\min }, T_{\max }\right]$ and making it dependent on the location of replication domains [16].

Acknowledgements. The author would like to thank A.Prusokas for a critical reading of the manuscript. 


\section{References}

[1] A. Baker, B. Audit, S. C. H. Yang, J. Bechhoefer, A. Arneodo. Inferring Where and When Replication Initiates from Genome-Wide Replication Timing Data. Phys. Rev. Let., 108 (2012), 268101, 1-5.

[2] A. Baker, C. L. Chen, H. Julienne, B. Audit, Y. dÕAubenton-Carafa, C. Thermes, A. Arneodo. Linking the DNA strand asymmetry to the spatio-temporal replication program I. About the role of the replication fork polarity in genome evolution. Europ. Phys. J. E, 35 (2012), 92, 1-25.

[3] A. Baker, H. Julienne, C. L. Chen, B. Audit, Y. dÕAubenton-Carafa, C. Thermes, A. Arneodo. Linking the DNA strand asymmetry to the spatio-temporal replication program II. Accounting for neighbor-dependent substitution rates. Europ. Phys. J. E, 35 (2012), 123, 1-12.

[4] J. Bechhoefer, N. Rhind. Replication timing and its emergence from stochastic processes. Tren. in Gen., 28 (2012), 374-381.

[5] E. Besnard, A. Babied, L. Lapasset, O. Milhavet, H. Parrinello, C. Dantec, J. M. Marin, J.M. Lemaitre. Unraveling cell type-specific and reprogrammable human replication origin signatures associated with G-quadruplex consensus motifs. Nat. Struct. \& Mol. Biol., 19 (2012), 837-844.

[6] E. B. Brodie of Brodie, S. Nicolay, M. Touchon, B. Audit, Y. d'Aubenton-Carafa, C. Thermes, A. Arneodo. From DNA sequence analysis to modeling replication in the human genome. Phys. Rev. Let., 94 (2005), 248103, 1-4.

[7] C. Cayrou, P. Coulombe, A. Vigneron, S. Stanojcic, O. Ganier, I. Peiffer, A. Puy, S. Laurent-Chabalier, R. Desprat, M. Mechali. Genome-scale analysis of metazoan replication origins reveals their organization in specific but flexible sites defined by conserved features. Gen. Res., 21 (2011), 1438-1449.

[8] C. Cayrou, P. Coulombe, A. Puy, S. Rialle, N. Kaplan, E. Segal, M. Mechali. New insights into replication origin characteristics in metazoans. Cell Cycl., 11 (2012), 658-667.

[9] Y. Clement, P. F. Arndt. Meiotic Recombination Strongly Influences GC-Content Evolution in Short Regions in the Mouse Genome. Mol. Biol. \& Evol., 30 (2013), 2612-2618.

[10] C. Costas, M. D. Sanchez, H. Stroud, Y. Yu, J. C. Oliveros, S. Feng, A. Benguria, I. Lopez-Vidriero, X. Zhang, R. Solano, S. E. Jacobsen, C. Gutierrez. Genome-wide mapping of Arabidopsis thaliana origins of DNA replication and their associated epigenetic marks. Nat. Struct. \& Mol. Biol., 18 (2011), 395-U190.

[11] N. Donley, M. J. Thayer. DNA replication timing, genome stability and cancer. Late and/or delayed DNA replication timing is associated with increased genomic instability. Sem. in Canc. Biol., 23 (2013), 80-89.

[12] A. P. S. de Moura, R. Retkute, M. Hawkins, C. A. Nieduszynski. Mathematical modelling of whole chromosome replication. NAR, 38 (2010), 5623-5633.

[13] M. dos Reis, L. Wernisch. Estimating Translational Selection in Eukaryotic Genomes. Mol. Biol. \& Evol., 26 (2009), 451-461.

[14] F. Gao, H. Luo, C. T. Zhang. DeOri: a database of eukaryotic DNA replication origins. Bioinformatics, 28 (2012), $1551-1552$.

[15] F. Gao, H. Luo, C. T. Zhang. DoriC 5.0: an updated database of oriC regions in both bacterial and archaeal genomes. NAR, 41 (2013), 90-93.

[16] M. G. Gauthier, P. Norio, J. Bechhoefer. Modeling Inhomogeneous DNA Replication Kinetics, PLoS one, 7 (2012), e32053 1-13.

[17] A. Gierlik, M. Kowalczuk, P. Mackiewicz, M. R. Dudek, S. Cebrat. Is there replication-associated mutational pressure in the Saccharomyces cerevisiae genome?. J. Theor. Biol., 202 (2000), 305-314.

[18] P. Green, B. Ewing, W. Miller, P. Thomas, E. Green. Transcription-associated mutational asymmetry in mammalian evolution. Nat. Gen., 33 (2003), 14-517.

[19] M. Hayashi, Y. Katou, T. Itoh, M. Tazumi, Y. Yamada, T. Takahashi, T. Nakagawa, K. Shirahige, H. Masukata. Genome-wide localization of pre-RC sites and identification of replication origins in fission yeast. EMBO J., 26 (2007), $1327-1339$.

[20] O. Hyrien, A. Rappailles, G. Guilbaud, A. Baker, C. L. Chen, A. Goldar, N. Petryk, M. Kahli, E. Ma, Y. d'AubentonCarafa, B. Audit, C. Thermes, A. Arneodo. From Simple Bacterial and Archaeal Replicons to Replication N/UDomains. J. Mol. Biol., 425 (2013), 4673-89.

[21] M. Kellis, B. W. Birren, E. S. Lander. Proof and evolutionary analysis of ancient genome duplication in the yeast Saccharomyces cerevisiae. Nature, 428 (2008), 617-624.

[22] M. L. DePamphilis. DNA replication and human disease. Cold Spring Harbor monograph series.

[23] G. I. Lang, A. W. Murray. Mutation Rates across Budding Yeast Chromosome VI Are Correlated with Replication Timing. Gen. Biol. \& Evol., 3 (2011), 799-811.

[24] A. C. Leonard, M. Mechali. DNA Replication Origins. Cold Spring Harbor Persp. Biol., 5 (2013), a010116, 1-18.

[25] I. Liachko, A. Bhaskar, C. Lee, S. C. C. Chung, B. K. Tye, U. Keich. A Comprehensive genome-wide map of autonomously replicating sequences in a naive genome. PLoS Gen., 6 (2010), e1000946, 1-12.

[26] L. Liu, S. De, F. Michor. DNA replication timing and higher-order nuclear organization determine single-nucleotide substitution patterns in cancer genomes Nat. Comm., 4 (2013), 1502, 1-10.

[27] M. Lundgren, A. Andersson, L. M. Chen, P. Nilsson, R. Bernander. Three replication origins in Sulfolobus species: Synchronous initiation of chromosome replication and asynchronous termination. PNAS, 101 (2004), 7046-7051.

[28] H. E. Luo, J. T. Li, M. Eshaghi, J. H. Liu, R. K. M. Karuturi. Genome-wide estimation of firing efficiencies of origins of DNA replication from time-course copy number variation data. BMC Bioinf., 11 (2010), 1-15. 
[29] J. Lygeros, K. Koutroumpas, S. Dimopoulos, I. Legouras, P. Kouretas, C. Heichinger, P. Nurse, Z. Lygerou Stochastic hybrid modeling of DNA replication across a complete genome. PNAS, 105 (2008), 12295-12300.

[30] M. Lynch. The origins of the genome architecture. Sinauer Associates Inc Publishers, Massachusetts.

[31] M. Lynch. Evolution of the mutation rate. Tr. Gen., 26 (2010), 345-52.

[32] A. T. McGeoch, S.D. Bell. Extra-chromosomal elements and the evolution of cellular DNA replication machineries. Nat. Rev. Mol. Cell Biol., 9 (2008), 569-574.

[33] M. Mechali, K. Yoshida, P. Coulombe, P. Pasero. Genetic and epigenetic determinants of DNA replication origins, position and activation. Curr. Op. Gen., \& Dev., 23 (2013), 124-31.

[34] C. A. Muller, C. A. Nieduszynski. Conservation of replication timing reveals global and local regulation of replication origin activity. Gen. Res., 22 (2012), 1953-1962.

[35] M. A. Nowak, H. Ohtsuki. Prevolutionary dynamics and the origin of evolution. PNAS, 105 (2008), $14924-14927$.

[36] S.Ohno. Evolution by Gene Duplication. Springer-Verlag, London.

[37] E. A. Pelve, A. C. Lindas, A. Knoppel, A. Mira, R. Bernander. Four chromosome replication origins in the archaeon Pyrobaculum calidifontis. Mol. Microbiol., 85 (2012), 986-995.

[38] T. J. Pohl, K. Kolor, W. L. Fangman, B. J. Brewer, M. K. Raghuraman. A DNA Sequence Element That Advances Replication Origin Activation Time in Saccharomyces cerevisiae. G3, 3 (2013), 1955-1963.

[39] B. D. Pope, D. M. Gilbert. The Replication Domain Model: Regulating Replicon Firing in the Context of Large-Scale Chromosome Architecture. J. Mol. Biol., 425 (2013), 4690-4695.

[40] J. G. D. Prendergast, H. Campbell, N. Gilbert, M. G. Dunlop, W. A. Bickmore, C. A. M. Semple. Chromatin structure and evolution in the human genome. BMC Evol. Biol., 7 (2007), 1-12.

[41] E. P. C. Rocha. The Organization of the Bacterial Genome. Ann. Rev. Gen., 42 (2008), 211-233.

[42] N. P. Robinson, S. D. Bell. Origins of DNA replication in the three domains of life. FEBS J., 272 (2005), $3757-3766$.

[43] C. C. Siow, S. R. Nieduszynska, C. A. Muller, C. A. Nieduszynski. OriDB, the DNA replication origin database updated and extended. NAR, 40 (2012), D682-D686.

[44] K. Skarstad, H. B. Steen, E. Boye. Cell Cycle Parameters of Slowly Growing Escherichia coli B/r Studied by Flow Cytometry. J. Bacteriol., 124 (1983), 656-662.

[45] O. Skovgaard, M. Bak, A. Lobner-Olesen, N. Tommerup. Genome-wide detection of chromosomal rearrangements, indels, and mutations in circular chromosomes by short read sequencing. Gen. Res., 21 (2011), 1388-1393.

[46] A. Srivatsan, A.Tehranchi, D. M. MacAlpine, J. D. Wang. Co-Orientation of Replication and Transcription Preserves Genome Integrity. PLoS Gen., 6 (2010), e1000810, 1-14.

[47] J. A. Stamatoyannopoulos, I. Adzhubei, R. E. Thurman, G. V. Kryukov, S. M. Mirkin, S. R. Sunyaev. Human mutation rate associated with DNA replication timing. Nat. Gen., 41 (2009), 393-395.

[48] T. Tatarinova, E. Elhaik, M. Pellegrini. Cross-Species Analysis of Genic GC(3) Content and DNA Methylation Patterns. Gen. Biol. \& Evol., 5 (2013), 1443-1456.

[49] E. Yaffe, S. Farkash-Amar, A. Polten, Z. Yakhini, A. Tanay, I. Simon. Comparative Analysis of DNA Replication Timing Reveals Conserved Large-Scale Chromosomal Architecture. PLoS Gen., 6 (2010), e1001011, 1-12.

[50] S. C. H. Yang, N. Rhind, J. Bechhoefer. Modeling genome-wide replication kinetics reveals a mechanism for regulation of replication timing. Mol. Syst. Biol., 6 (2012), 404, 1-13.

[51] C. C. Weber, C. J. Pink, L. D. Hurst. Late-Replicating Domains Have Higher Divergence and Diversity in Drosophila melanogaster. Mol. Biol. \& Evol., 29 (2012), 873-882.

[52] P. Worning, L. J. Jensen, P. H. Hallin, H. H. Staerfeldt, D. W. Ussery. Origin of replication in circular prokaryotic chromosomes. Env. Biol., 8 (2006), 353-361.

[53] Z. Wu, H. Liu, Hailong, J. Liu, X. Q. Liu, H. Xiang. Diversity and evolution of multiple orc/cdc6-adjacent replication origins in haloarchaea. BMC Gen., 13 (2012), 1-16. 